\title{
Integrated ICT framework for Distribution Network with Decentralized Energy Resources: Prototype, Design and Development
}

\author{
N. Hadjsaid, Senior Member IEEE, L. Le- Thanh, Member IEEE, R. Caire, Member IEEE, \\ B. Raison, Member IEEE, F.Blache, B. Ståhl, R. Gustavsson
}

\begin{abstract}
Electrical Distribution Systems (EDS) are facing ever-increased complexity due, in part, to fast growing consumer demands and the integration of large amounts of distributed energy resources. The conventional operation modes need to be adapted to the significant changes. With advanced Information and Communication Technology (ICT) infrastructure and sophisticated new functionalities for energy management, "Smart Grid" is becoming the most forward-looking solution to address these facing challenges. Having initiated a conceptual model, architecture, and implementation plan for the system in previous European research projects (CRISP [1] and MICROGRID [2]). A new integrated platform for ICT based distributed control and local management is currently being designed, with the goal of demonstrating the feasibility of these new concepts. The overarching design of coordination and control between the real automation devices and power system components will be carried out with an analogical micro network ( $\mu$ Grid) including real power operation components such as RTUs. Therefore, some high level functions relied on advanced ICT system such as fault management; voltage control and adaptive reconfiguration are developed and tested for this purpose.
\end{abstract}

Index Terms- Active distribution networks, ICT and Agent Based Systems evaluation, Distribution Network, Distributed DMS, Restoration process, Distributed Generators, Protection devices.

\section{INTRODUCTION}

$\mathrm{D}$ istribution grids are facing tremendous challenges due to several factors. Among them, the most important is the increasing penetration of Distributed Energy Resources (DER) especially those based on Renewable Energy Sources (RES). These changes are expected at several levels and scales from the generation and commercialization businesses to the distribution grid operation. As such Electrical Distribution Systems will require new planning strategies and tools, new design methodologies, redefining operation and control of

N. Hadjsaid, L. Le-Thanh, R. Caire and B. Raison are with IDEA (a joint research center between EDF, Schneider Electric and Grenoble Institute of Technology) and Grenoble Institute of Technology, BP 46, 38402 Saint Martin d'Hères, France (phone: +33 (0) 476827152; e-mail: Nouredine.Hadjsaid@g2elab.grenoble-inp.fr)._Björn Ståhl and Rune Gustavsson are with Blekinge Institute of Technology (BTH), Sweden. This work was funded partially by the European Commission under the $6^{\text {th }}$ framework program number FP6-038576 call FP6-2005-TREN-4. electrical networks for instance. Currently, there are a large amount of studies and initiatives to assess future functions of intelligent power systems and to construct a hybrid, fundamentally different architecture for an ICT-network enabling the power grid to flexibly accommodate novel devices or clusters of devices. Most noticeable in this respect is the Intelligrid [3], a program in the United States executed by EPRI. The scope of Intelligrid is very broad and not directly focused on the intake and flexible accommodation of DG-RES. A project with a comparable theme is Gridwise [4]. A technical summary of architectural issues has been given recently as a final report on architectures within the CRISP project [5]. Other important relevant initiatives on the European level are the EU_DEEP-project [6] and the activities performed on a European coordinated scale in the IREDcluster [7].

As described in various articles in literature (e.g. [8]) there are many implications for the grid when making a transition from centralize to decentralize control with merely some central coordination. When compared to hierarchically operated electricity grids with power centrally generated at high voltage levels on a large scale delivering electricity to consumers on lower Voltage levels in the network, Smart Grids [9] offer a number of challenges for technological research. Indeed, the EU long-term vision on future grids, European Smartgrids Technology Platform waterfall grid models will be more and more replaced by grids with electricity produced via installations or clusters of installations 'bubbling' upwards. ICT is considered to be an essential enabler for this new development. Grids with components connected by modern communication technology, Smart Grids equipped with facilities for more intelligent coordination, are expected to play an ever more pronounced role in such a transition.

On the same ambitious research direction, the INTEGRAL project (Integrated ICT- platform based Distributed Control (IIDC) in electricity grids with large share of Distributed Energy Resources and Renewable Energy Sources), will push the investigations further. Practical validations of INTEGRAL project rely on three different field tests which cover a wide range of different conditions including: normal, critical and emergency operations. All these demonstrators should show the advantages and the robustness of agent based coordination for network devices and DER/RES sharing, in a common 
framework, a cost effective and reliable ICT architecture. This architecture is philosophically a bottom-up process and the sizing of such concept has to be assessed. Various actors, such as grid operator or DER owners, will get benefits of such common ICT infrastructure [10].

This panel paper presents a test bench to validate the bottom up control command process in an active distribution network with controllable load and dispatchable DER. The test bench, which is based on a reduced scale distribution network [11] including the real electrical components and an integrated ICT system, allows the researchers to validate every research results with an industry quality reference. Moreover, it is also a valuable resource to train engineers to meet the closest requirements of the new energy industries.

The following section describes the general local control and management system prototype for this test bench. Then, as part of that prototype, the ongoing demonstration which aims to facilitate self-healing functionality in emergency operating mode of distribution system with DER is presented. The analogical $\mu$ Grid network's reduction and configuration as well as the role of modules layers, local agent and supervisor are discussed. Finally, a deployment scheme for the ICT integrated system, which makes it possible its validation is suggested.

\section{Control AND MANAgEMENT PRototype For TeSt BENCH}

A prototype has been developed in order to have the overall system requirement deployed in the $\mu$ Grid. Figure 1 portrays the simplified system prototype.

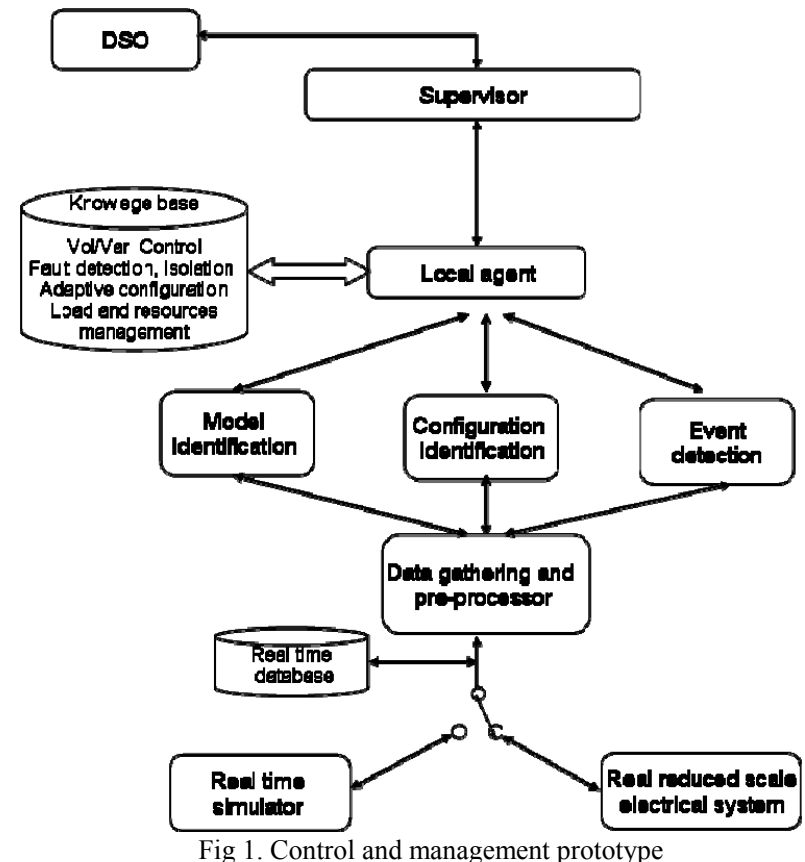

Real time data measured directly with real RTUs (Remote Terminal Units) from $\mu$ Grid or a real time simulator are received by the data gathering and pre-processor modules. These modules preprocess the data by removing undesired discrepancies data and verifying the abnormal values. Processed data are stored in a distributed real time database for logging and other purposes, and is sent to the higher level module for further processing. System components like: electrical lines, transformers, and types of DER are identified in the "Model Identification" module. The "Configuration Identification" module determines the actual configuration of $\mu$ Grid by analyzing the availability of components, state of circuit breakers and switches. A variation in the operating point or an abnormal change in data is studied in the "Event Detection" module. It determines the nature and the seriousness of event and alerts the local agent. Then all processed data from these modules are sent to local agent.

It assesses the logical behaviors of the systems and finds solution relying on a knowledge base which has been developed in IDEA/G2ELab from the work of many researchers in electrical distribution system with presence of DER/RER such as vol/val control [12]; stability and preventive control [13][14] fault detection [15], optimization reconfiguration [16] and so on.

When the scenarios are determined, the local agent gives to a supervisor (autonomic manager) a solution for its decision. For every event that occurs, the Distribution System Operator (DSO) should be also notified. And in crucial situation, DSO can also give order for own process execution.

Using above prototype, the first test bench with selfhealing functionalities is being implemented for the $\mu \mathrm{Grid}$ within the framework of INTEGRAL project. Self-healing functionality includes fault detection and isolation, then adaptive reconfiguration for service restoration [11]. The design of different layer, agent as well communication system is described in the following sections.

\section{ANALOGICAL $\mu$ GRID CONSTRUCTION AND INTEGRATED ICT SYSTEM DESIGN}

The analogical micro distribution network (figure 2) was sized by aggregating some electrical nodes of a real distribution network having $30 \mathrm{MVA}$ rated power at $20 \mathrm{kV}$. In order to best represent the behavior of a real network for many RTUs, and satisfy economical conditions, the network of test bench of $30 \mathrm{kVA}, 0.4 \mathrm{kV}$ was adopted.

The reduced scale of the $\mu$ Grid components was carried out through different ratios (power, inertia and voltage) to assure the similarity of the internal static and dynamic behaviors of the network which includes aggregated loads, network components (on-load tap changers for instance) and DER/RES. Indeed, the performance of the control systems must be unchanged in comparison with the real system. This network has 14 nodes, 17 lines, 10 loads and 6 DERs, divided into several areas which represent different network characteristics. For instance, the electrical lines are generally underground cables in urban areas and overhead lines in rural. Otherwise, operation criteria differ in the various networks such as critical clearing time for the circuit breakers and the switches. 


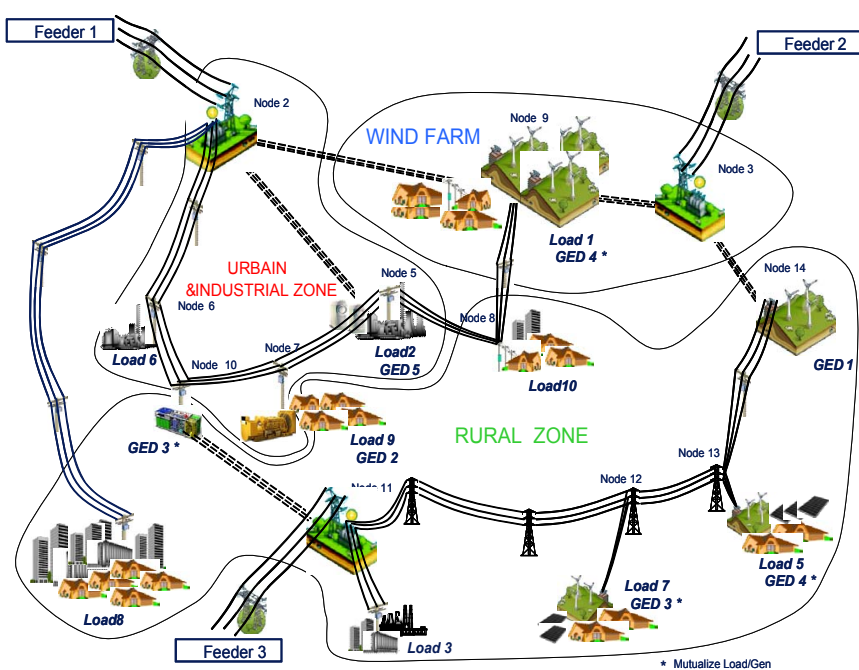

Fig 2. Topology of the analogical distribution $\mu$ Grid

To better demonstrate the self-healing functionalities within the analogical $\mu$ grid, we propose that communicating automation devices are interconnected within the demonstration site, forming a Local Area Network (LAN). Within a $\mu$ Grid (substation) or operating center (control room) a LAN may be used to provide high-speed reliable communications between local equipment as well as via routers to nearby or remote LANs, or to wide-area networks (WANs) such as the Internet..

A simplified example of the ICT architecture for selfhealing demonstration is presented in Figure 3. As the fault location algorithm has been developed within MATLAB the local agent were built within MATLAB and MATLAB OPC toolbox. OPC Data Access standard [17] via Ethernet provides the common protocol for communication between OPC server associated with communicant RTU (Modbus/TCP) or with SCADA center and OPC client (MATLAB OPC Toolbox) playing a role as local agent.

Communicating remote terminal unit (RTU) such as fault recorder emulator developed within Labview [18], fault indicator (Flair 200C) are connected within several LAN. These devices communicate with other PCs and agents using an 'Object link embedding for Process Control' (OPC) client/server solution. Finally, the advanced control and batch execution to accomplish self-healing functionalities could be carried out directly from local agent or indirectly from DSO (with the local agent advices).

The data communication on the agent levels and below has to be first specified then tested to ensure that all desired functions are accounted for. Because LANs and their respective communication technologies impose limits on data exchange performance, an experimental communication network environment was built during the CRISP project. This environment allowed for controlled experimentation on performance parameters such as bandwidth and latency, for communication that crossed LAN boundaries [5].

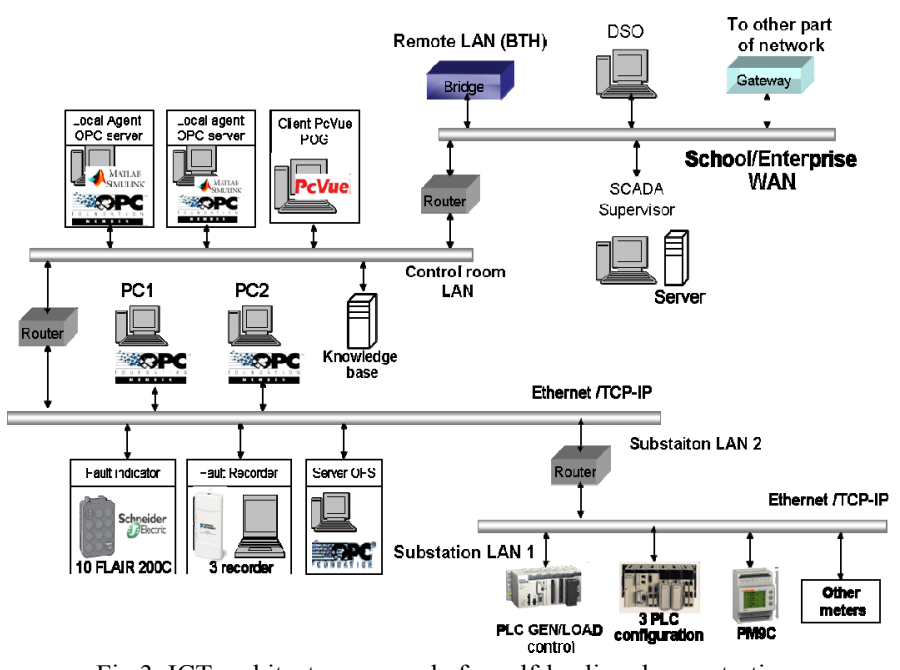

Fig 3. ICT architecture example for self-healing demonstration

\section{CONCLUSIONS}

The transition from current to future EDS while accommodating large amount of DER and keeping the desired level of quality and reliability will require investigations on decentralized operation as well as on adaptive architectures. This panel is dedicated to some of the concepts under development at Grenoble Institute of Technology and IDEA dealing with these decentralized operating modes including emergency management functions. These concepts are being developed jointly with an experimental platform (the $\mu$ Grid) setup in Grenoble Institute of Technology. This process is intended to validate the above concepts and to size correctly the needs in term of communication and computerization performances.

\section{REFERENCES}

[1] G.J. Schaeffer, H. Akkermand, et Al. "Final Summary Report", Deliverable D5.3, 2006, http://crisp.ecn.nl/deliverables/D5.3.pdf

[2] A. Dimeas, N. Hatziargyriou, "Agent based Control for Microgrids" Power Engineering Society General Meeting, 2007. IEEE 24-28 June 2007 Page(s): 1 - 5

[3] The CEIDS and Intelligrid vision is explained following the link: http://www.epri.com/IntelliGrid/

[4] Gridwise project: A complete description of the initiative can be found at: http://www.gridwise.org/

[5] Ch. Andrieu et. Al. Distributed network architectures, Deliverable D1.7, 2006. http://www.ecn.nl/crisp/deliverables/D1.7.pdf

[6] EUDEEP project: the website of this project can be found at: http://www.eudeep.com/. In the project a thorough classification of load types has been given.

[7] IRED project: on the conference website the conference program can be found: http://www.2ndintegrationconference.com/agenda.asp

[8] Mohamed Choukri BenHabib, Jorge Duarte, TU/Eindhoven, Maarten Hommelberg, René Kamphuis, Cor Warmer, ECN, Flexible electricity grids Power Electronic System and ICT-requirements for novel electricity distribution grids, 2006

[9] European Smartgrids Technology Platform, Vision and Strategy for Europe's Electricity Networks of the future. EUR22040, 2006.Electricite de France, " Le Micro Réseau : 30 ans d'activité" Direction des Etudes et Recherches, Departement FCR, 1986

[10] Integrated ICT-platform based Distributed Control in electricity grids with a large share of Distributed Energy Resources and Renewable Energy Sources - Deliverable D2.1 - "High Level Specification of the Functionality for novel electricity distribution grids."

[11] L. Le-Thanh, R. Caire,, B.Raison, S. Bacha, F. Blache, G.Valla :"Test Bench for Self-healing Functionalities applied on Distribution Network 
with Distributed Generators", PowerTech 2009, 28June-2 July 2009 , Bucharest, Romania

[12] O. Richardot, A. Viciu, Y. Besanger, N.Hadjsaid, Ch. Kieny, "Coordinated Voltage Control in Distribution Networks Using Distributed Generation", PES TD 2005/2006 Page(s):1196 - 1201

[13] L. Le-Thanh, T. Tran-Quoc, O. Devaux, O. Chilard, Ch. Kieny, N. Hadjsaid, J.Cl. Sabonnadiere, "Dynamic behaviors of Distributed Generators and Proposed Solutions to Avoid Loss of Critical Generators", Power Engineering Society General Meeting, 2007. IEEE 24-28 June 2007 Page(s): 1 - 6[1]

[14] L.Le-Thanh, JC. Sabonnadiere, Tran Quoc Tuan, O. Devaux, O. Chilard, C.Kieny, N. Hadjsaid, "Hybrid methods for transient stability assessment and preventive control for distributed generators", IEEE PES General Meeting, Pensylvania, USA, July 2008

[15] Delcho Penkov. "Fault location in electrical MV networks with Distirbuted Generators." Doctoral Thesis - Grenoble Institut of Technology, 2006

[16] B. Enacheanu, B Raison, R Caire. "Radial Network Reconfiguration Using Genetic Algorithm Based on the Matroid Theory." IEEE Transaction on Power System Vol. 23, No. 1, pp 186-195, 2008

[17] OPC Foundation, "Dedicated to interoperability in automation" http://www.opcfoundation.org/

[18] National Instrument - Labview http://www.ni.com/labview/

[19] I. Munteanu, A. I Bratcu, S. Bacha, D. Roye, J. Guiraud "Hardware-inthe-loop Simulator for a Class of Variable-speed Wind Energy Conversion Systems: Design and Performance Assessment", IEEE Transactions on Energy Conversion, to be published

\section{BIOGRAPHIES}

Nouredine HADJSAID (SM'06) received his Ph.D. degree in Electrical Engineering and "Habilitation à Diriger des Recherches" degree from the Institut National Polytechnique de Grenoble (INPG) in 1992 and 1998, respectively. From 1988 to 1993, he served as a research and teaching assistant at the Ecole Nationale Supérieure d'Ingénieurs Electriciens de Grenoble (ENSIEG) and at the Laboratory d'Electrotechnique de Grenoble (LEG). He is a full professor at Grenoble Instutute of Technology and a General Director of a common research center on future electrical distribution system between EDF, Grenoble Institute of Technology and Schneider Electric.

Luong LE-THANH (M'08) received his Diplôme d'Etudes Approfondies (DEA) and Doctorat de l'INPG degrees from the Institut National Polytechnique de Grenoble (INPG) in 2004 and 2008 , respectively. He is now working as a fellow researcher in the GIE-IDEA (FRANCE). His research interests are the power systems equivalent, power system stability and distributed generators' impacts in electrical distribution system.

Raphael CAIRE (M'04) received his Diplôme d'Etudes Approfondies (DEA) and Doctorat de l'INPG degrees from the Institut National Polytechnique de Grenoble (INPG) in 2000 and 2004. He had been working in Power Electronic field, in USA at the Center of Power Electronic System (CPES) in 2000 and within several EDF research centers in Germany and in France from 2004 to 2006. He is now associate professor at Grenoble Institute of Technology (Grenoble-InP) at the Ecole d'ingénieurs en énergie eau et environnement (ENSE3) in the Grenoble Electrical Engineering laboratory (G2Elab). His research is centered on the impacts, production control of dispersed generation on distribution system and critical infrastructures.

Bertrand RAISON (M'03) was born in Béthune, France in 1972. He received his M.S., Ph.D. and "Habilitation à Diriger des Recherches" degrees in electrical engineering from the INPG, France, in 1996, 2000 and 2007, respectively. He has joined since 2001 the Grenoble Electrical Engineering laboratory (G2Elab) and the Ecole d'ingénieurs en énergie, eau et environnement (ENSE3) as an associate professor. His general research interests are fault detection and localization in electrical systems. He is, since 2009, a full time professor at the Université Joseph Fourier in Grenoble.
François BLACHE received his $\mathrm{Ph}-\mathrm{D}$ in Electrical Engineering from « Institut National Polytechnique de Grenoble » (France) in 1995. He joined this institute in 2000, to work as Research Engineer in the Power System Group of the " Grenoble Electrical Engineering laboratory » (G2Elab). At present, he is in charge of Real-time hybrid simulation projects.

Björn STÅHL got his M.Sc in computer science at Blekinge Institute of Technology (BTH) in 2006, and is currently a Ph.D. student at BTH. His research focus and areas of interest concern monitoring, virtualization and dynamic instrumentation- of live software intensive systems, primarily within the context of resilience in critical information infrastructures.

Rune GUSTAVSSON is a professor in computer science at Blekinge Institute of Technology (BTH). Gustavsson has been appointed to several assignments as scientific advisor to international and national research funding organisations. Gustavsson has publications in books, journals and proceedings in his areas of interest that include; resilient critical infrastructures, ICT support of future Smart Grids and service oriented computing. 